International Journal of

Advanced Science and Convergence

\title{
Study on the Storage Virtualization Technology for Smart Work Service in Mobile Environment
}

\author{
Ssang-Hee Seo ${ }^{1}$ and Bong-Hyun Kim ${ }^{2}$ \\ ${ }^{1}$ Department of Computer Science and Engineering, Kyungnam University, Gyeongnam, CO 51767, Korea \\ ${ }^{2}$ Department of Computer Engineering, Seowon University, Chungbuk, CO 51767, Korea
}

\begin{abstract}
Background/Objectives: As the paradigm changes with technological advancement and information society, next-generation products that can access information anytime, anywhere through mobile Internet devices through Smart work are being released. In particular, in the coronavirus era, there is a lot of interest in the introduction of Smart work. Methods/Statistical analysis: Companies that only pursued work in the office tend to support a free and creative work environment that goes beyond temporal and spatial restrictions. In addition, we are hoping for collaboration, efficiency, and organizational innovation through smart work communication. The introduction of Smart work by companies can be expected to have a positive impact on companies. Findings: Therefore, in this paper, we intend to provide a safe Smart work service through research and application of storage virtualization technology. In other words, a Smart work service was designed by securing the technology for operating the latest operating system based on mobile virtualization technology and storage virtualization technology, which is the core of mobile security. Improvements/Applications: The storage virtualization-based mobile work service proposed in this paper can be used for mobile terminals and special security-enhanced terminals that require high security in mobile environments. In addition, it can be used for mobile financial services for safe payment/payment such as electronic payment and internet banking, and prevents corporate information leakage and illegal access by unauthorized users in work environments such as Smart work using mobile terminals.
\end{abstract}

\section{Index Terms}

Smart work, Storage virtualization, Cloud resource, Mobile device management, Mobile environment

\footnotetext{
Corresponding author : Bong-Hyun Kim

bhkim@seowon.ac.kr

- Manuscript received June 25, 2020.

- Revised July 30, 2020 ; Accepted September 21, 2020.

- Date of publication September 30, 2020.
}

(c) The Academic Society of Convergence Science Inc.

2619-8150 ( 2020 IJASC. Personal use is permitted, but republication/redistribution requires IJASC permission. 


\section{INTRODUCTION}

The present age is the information age, and the rapid development of information and communication technology is making a big difference in our daily life. We are entering an era in which the intangible resource of information excels in monetary value, and this change has increased the desire for use of PC users. As the paradigm shifts toward technological development and information society, in recent years mobile internet devices through Smart work Next-generation products that can access information anytime, anywhere are being released[1]. In an era of rapid changes in technology and trends, innovation of work space also emerged as a new topic as promptness and creativity of work became more important than anything else. We live in an era where people do not work but work with people. We live in a society where Smart work is implemented, that is, a knowledge-based society. As network environments and IT technologies are advanced worldwide, and interest in low-carbon green growth using IT is increasing, Smart work is being actively promoted in major developed countries. In the $21 \mathrm{st}$ century amid rapid IT technology development, the modern society has brought about the use of smart mobiles due to the development of mobiles, and has made people into a single object into the network. In this situation, the work space has been continuously developed so that people can perform work creatively and freely, and it has become a society in which a work environment can be created comfortably without time and space constraints[2-3]. In addition, the concept of Smart work emerged as a new concept of working in a space prepared outside the office instead of working as a kind of remote work such as home work.

In other words, it is a term that refers to performing work outside the office using information and communication technology without going to the office. It can be defined as a flexible working form that is not restricted to the place or time by deviating from the method of working at a fixed time in the past. In the past, efforts have been made to attempt to work from home or remotely, but it has not been able to bring about a major policy and social direction due to the efforts to implement it in terms of technology. However, if you look at the background of the rise of Smart work in the industry today, it can be said that it is stronger than before[4]. One of the backgrounds is the development of IT technology. In the implementation of Smart work, the advancement of ICT technologies such as wireless networks and VoIP that enable remote work outside of companies and the development of digital devices such as tablet PCs and smart phones. It is a prerequisite[5-6].

Smart work is a future-oriented work environment that enables you to efficiently process work anytime, anywhere in various places and mobile environments, rather than the traditional office concept, which is a designated work space, and improves work speed and productivity through rapid work processing in the field. In addition, real-time collaboration through remote collaboration enables rapid decision-making and quick problem solving[7]. The flexibility of working types can expect positive effects, such as expanding employment opportunities for the underprivileged.

Smart work can be said to be a form of work that uses information and communication technology to have a continuous relationship with the people involved in performing work without restriction of time and place. Smart work is a job concept with deeper flexibility in terms of time and place, and it means seeking to improve job efficiency through the use of various types of information and knowledge, and mutual trust and cooperation. In addition, Smart work can be classified into types such as homework, Smart work center work, and mobile mobile work based on the flexibility of time and place. This is used conceptually similarly to terms such as teleworking, telecommuting, and flexible working.

The introduction of Smart work by companies can be expected to have a positive impact on companies such as cost reduction, work productivity improvement and customer satisfaction increase, excellent talent acquisition and utilization, and organizational expertise enhancement. With the introduction of Smart work, a large amount of information can be exchanged at a high speed, so most tasks such as personal contact and document sharing can be handled with Smart work. You can open it anytime, anywhere. In particular, the introduction of Smart work enables quick acquisition of information and real-time communication, overcoming time and space constraints, and even improving work speed and efficiency, so many organizations are making efforts to use smart devices for work. As such, the spread of smart devices can be seen as a new paradigm that has a great influence on our office environment and the business activities of the organization and further leads change. In addition, the Smart work environment is affecting various parts of the organization such as manpower management and work type, and the Smart work environment is not just a means to solve the limitations of time and space. Ultimately, it is meaningful to actively respond to environmental changes by inducing their creativity and innovative thinking and actions through the working environment for employees[8-10].

At a time when interest in Smart work is growing, it is necessary to conceptually clarify the definition of Smart work along with the characteristics of Smart work. Smart work does not consist of simple introduction and use of Smart work devices. In 
addition, Smart work is based on remote collaboration, not simple remote work. It refers to a form of collaboration based on information and communication technology[11]. And, it is a technological facility base that allows you to work regardless of time and place. Smart work allows you to work anytime and anywhere without time and space constraints. Lastly, Smart work refers to a change from work hard to work smart. However, the existing Smart work method uses MDM as the most basic security measure. There are still many problems with respect to how to protect data when malicious codes are planted in a smartphone. Therefore, in this paper, we intend to provide a safe Smart work service through research and application of storage virtualization technology. In other words, a Smart work service was designed by securing the technology for operating the latest operating system based on mobile virtualization technology and storage virtualization technology, which is the core of mobile security.

\section{SMART WORK ENVIRONMENT}

First, Mobile office, the representative environment of Smart work, refers to a business system that can handle company work from outside through a mobile terminal (mobile communication device) anytime, anywhere. It consists of mobile terminals such as mobile office Smart work, solutions such as business related apps (APPS), mobile communication networks such as WCDMA and WIFI, and mobile platforms[13].

Smart work, a representative terminal of mobile offices, is a multi-cell phone with small computer functions that can be used not only for calling, but also for e-mail, Internet, and e-books. Furthermore, it can be expanded to the concept of Smart Mobile. This means a mobile terminal and service that accurately selects the information desired by the user based on the situation and provides it quickly and conveniently. Through the mobile office, work efficiency can be improved, public/customer service reinforced, vertical and horizontal communication can be strengthened, and cost reduction effects can be expected[14]. In addition, Smart work has an intuitive user interface, quick response speed, multipoint touch, and an open application service model, enabling free installation and execution of various applications.

Mobile $\mathrm{O} / \mathrm{S}$ has various functions and advantages and disadvantages depending on its type. In the case of iPhone, it is user-friendly and operates the industry's largest application store, but it is inconvenient because it adheres to a closed and exclusive policy, while Android has an advantage of excellent openness. However, management costs such as development/operation costs and risks are high according to multiple terminals. As for the operating system (OS) of PC, MS Windows accounts for more than $90 \%$, while in mobile environment, there are various such as Android, Windows Mobile, iPhone OS, and Blackberry OS. Along with the abolition of the WIPI mandate, various brands and OS-based Smart work have been spread in earnest in Korea. Such a mobile server platform should support various terminal environments and provide functions that can be applied to various Smart work environments at low cost. In order to implement concepts such as 'One Source Multi-use' or 'Any Device Any Platform', it supports various client types such as Smart work platform type, mobile web, mobile application, and rich client, and provides an interface based on user experience.

Second, it is working from home. Home work is a flexible form of work in which a work space is provided at home using information and communication technology, and facilities and equipment necessary for work are built. To work from home, establish the same work environment as the office and use security authentication technology to access the company's intranet to perform work, or to collaborate with other workers away from the headquarters or remote locations, such as video conferencing and sharing work processes. I also go to practice. In other words, it refers to a work environment in which the main computer of the workplace and the computer installed at home are connected through an information and communication network to handle work at home without going to work. With the development of information and communication, the working type that requires a certain amount of space and time, like the conventional office type, has changed greatly, and it is possible to work from home in which all contact information is performed at the necessary time at home while using computer communication. These telecommuting services include a method in which a company is equipped with a job infrastructure (In-House type) and a method of receiving services using a telecommuting platform provided by a specialized company (Hosting type). The platform and PC environment necessary for working from home are provided as essential items, and the remaining elements such as supplementation and collaboration are optionally provided. In addition, it is possible to use enterprise solutions (groupware, ERP, tax/accounting solutions, etc.) for SMEs.

Third, it is a Smart work center. The Smart work center is a form of working in an environment similar to an office using IT infrastructure in a dedicated facility built near the employee's residence. The smart work Center is a facility equipped with an IT-based remote work system to reduce the amount of time spent in offices in the city center and to work 
near the residential area of workers. It is a complex space that provides the office environment necessary for knowledge work activities. It provides the same working environment as described above, so it is easy to engage in work and manage service. The environment of the Smart work center consists of facility infrastructure, IT infrastructure, and operation infrastructure. The facility infrastructure is a facility that composes the physical environment, and includes a work space used for working with workers, a conference room for online and offline meetings, seminars, education, etc., a cultural space for relaxation, and peripheral devices for work. It can be divided into a common office space that can be used jointly. Smart work centers can rent real offices and are equipped with video conferencing, highspeed Internet, telephone systems, fax machines, printers and computer systems. In addition, staff are prepared for professional secretarial services and various interpretation and translation services[15-17]. The Smart work center application infrastructure provides applications (collaboration and communication, etc.) so that remote users can work in the same environment as the company from outside. Virtual company intranet, web disk, schedule management, collaboration (video conferencing UC, etc.), web mail, office programs, etc. For the effective use of the Smart work center, the building and operating entity should utilize the operation management system. In general, an infrastructure for operation management such as facility management, information desk, and access check is established so that workers can conveniently reserve and use various facilities of the Smart work center.

As green growth emerges as the future vision of companies along with the coronavirus era, one of the efforts of companies to reduce carbon dioxide emissions is to reduce the emission of carbon dioxide generated during travel, such as commuting and business trips, and energy savings by improving the working method. Smart work is being spotlighted as an alternative to converting to a low-carbon work environment.
At the same time, recent changes in social and cultural perceptions are also contributing to the spread of Smart work. As workers' values for work change from work life-centered to work \& life balance that values personal life and quality of life, the demand for Smart work that makes it easy to have time with leisure and family such as selfdevelopment time is increasing. In addition, people's perceptions of work are changing from the unit of time and space' to the unit of performance'. Therefore, when the Smart work is introduced, the practical trust that the work will be completed is formed rather than the expectation that the worker will work for a specific time, thereby creating a cultural environment that facilitates the introduction of Smart work.

In addition, it creates the workforce value of Smart work for vulnerable people such as women, the elderly, and the disabled. In addition, there are expected effects from the introduction of Smart work, such as reduction of business operation costs due to the reduction of office space of the government and companies, improvement of productivity due to remote work, and improvement of employee satisfaction. These are the main factors that actively promote the adoption of Smart work by both the government and enterprises. In this way, Smart work does not simply replace basic tasks with mobile, but uses the characteristics of mobile to improve processes and create new values.

The Smart work development stage is the first stage, where communication between employees is activated across the boundaries of time and place. In the second step, transparent information sharing is realized through real-time linkage with the in-house business system. In three stages, the way you work itself changes. Such changes are expected to have a great impact on companies belonging to general manufacturing and service industries as well as related industries such as mobile communication and IT. Figure 1 shows the characteristics of the environment changed due to the introduction and application of Smart work.

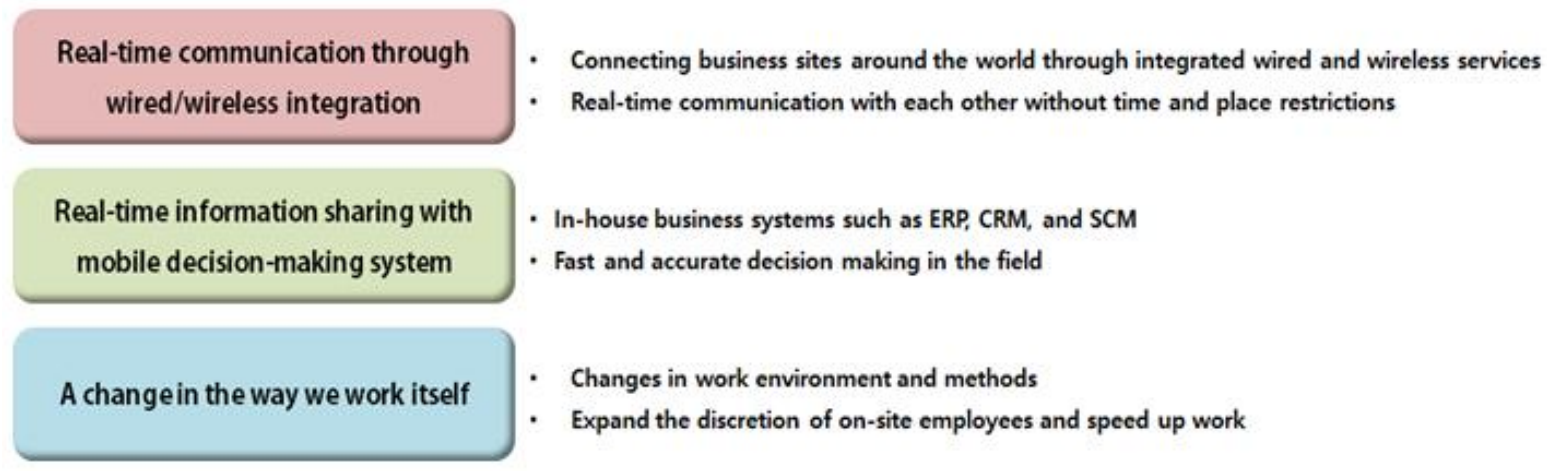

Fig. 1. Characteristic of smart work environment 


\section{SMart Work AND Mobile OfFICE TRENDS}

The introduction of Smart work has various expected effects at the level of not only companies, but also workers and countries. At the corporate level, cost reduction and performance improvement have been demonstrated mainly by large companies and foreign companies. Representatively, IBM reduced office space and operating costs by $40-60 \%$, and improved employee productivity. From the perspective of workers, the reduction in commuting time increases personal life and quality of life, resulting in higher work satisfaction, as well as career breaks and lower turnover rates. At the national level, there is an expectation effect of reducing traffic congestion due to commuting and dispersing the concentration of offices in the city center, and there is an advantage that can provide a tip for solving low birthrate and aging population.

In the domestic and private sectors, the rate of use of Smart work in 2015 was $14.2 \%$, an increase of $3.8 \%$ p compared to 2012 . The Smart work utilization rate is higher as the size of a company increases. Among the Smart work types, mobile offices $(20.1 \%)$ are the most used, followed by teleconferences $(11.2 \%)$, telecommuting $(9.2 \%)$, and Smart work centers $(5.9 \%)$. On the corporate side, Smart work was found to be effective in reducing office operation and management costs $(59.5 \%)$ and working hours $(54.9 \%)$. In addition, on the personal side, it was found that it is effective in reducing commuting time and expenses (73.2\%), and securing parenting and housekeeping time (63.6\%). In the case of female workers, it is recognized that Smart work is helpful in securing parenting or housework time $(64.4 \%)$, and by age, $60 \%$ of those in their twenties saw that it helps relieve low birthrate[13-15]. Most of the public sector workers had experience using Smart work centers $(85.8 \%)$. The most common reason for using a Smart work center was to save commuting time (71.0\%), and when using a residential Smart work center, it was found that more than one hour $(45.7 \%)$ compared to office commute. It was found that working at a Smart work center is effective in increasing efficiency $(80.1 \%)$ and reducing working hours $(79.5 \%)$ in terms of work. In particular, $60 \%$ of respondents said that their productivity (over 30\%) has increased. Figure 2 shows the frequency of use by Smart work service type. In addition to the introduction of Smart work, there are increasing cases of introducing mobile offices to increase business efficiency. In addition, as the existing PC-oriented Smart work environment expands to mobile terminals such as smartphones and tablet PCs, related security issues are rapidly rising as the use of wireless networks increases. In particular, as mobile offices are activated, the risk of data leakage and hacking using wireless networks increases due to loss of terminals. In addition, malicious code infection caused by reckless application use is also pointed out as a major security threat[17-19].

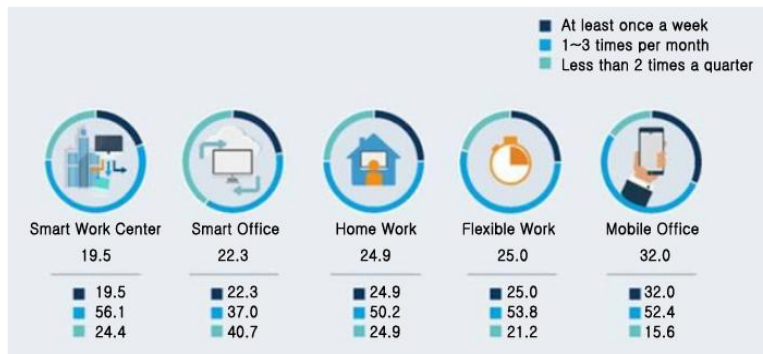

Fig. 2. Frequency of use by smart work service type

Therefore, a mobile terminal management (MDM) solution that enables a mobile terminal to be used in a safe environment is being used when introducing a Smart work. Globally, Sybase, a strong player in the MDM market, mobile iron, and genprize are applying security technology in the Smart work platform market based on the know-how accumulated in the global market. Although competition in the MDM market is hot, it is difficult to find a particularly large difference by looking at the product introduction data of each company. Application management, asset management, and resource limiting are commonly supported by various MDM solutions, and the functions of most MDM solutions are not significantly different.

Mobile offices, which occupy a large proportion of Smart work types, are most commonly used for mobile mobile work, but there is a problem of poor security. As mobile devices begin to emerge as a major target for hacking, it is time to prepare countermeasures. However, the level of domestic preparation for this is still insufficient. Currently, according to domestic mobile carriers and security industry officials, most of mobile security is in the form of supplying exclusive vaccines through app marketplaces. It mainly analyzes the patterns of newly generated malicious codes overseas, updates the mobile-only vaccine, and takes management measures if necessary.

All three major mobile telecommunication companies in Korea are operating Google Androidbased app marketplaces, and since they do not have separate mobile security policies, they do not have any other means other than downloading and installing vaccines by customers. In addition, as the opinions of major mobile security technology users, including mobile operators, are so, domestic security companies have only thoughts other than vaccines, but do not have the technology to respond to mobile security threats in various fields. In the future, the trend of the mobile security market should go 
beyond MDM, which acts as a vaccine and a kind of firewall, to manage mobile application distribution rights. In addition, like a web app in a PC environment, other apps other than the basic operating system (OS) will be managed through a separate virtual environment (VPN, virtualization technology). As mobile devices are starting to emerge as major hacking targets, it is necessary to prepare countermeasures, but the level of preparation in Korea is still insufficient. MDM is used as the most basic security measure, and there are still many problems regarding how to protect data when malicious code is planted in a smartphone.

Therefore, in this paper, it was determined that it was necessary to solve the security problem for the safety of the Smart work service, and the research and application of mobile virtualization technology was conducted. Therefore, research was conducted to secure a technology for operating the latest operating system based on mobile virtualization technology and storage virtualization technology (mobile cloud resource management technology), which is the core of mobile security.

\section{Storage Virtualization as a SMART WORK SERVICE}

Storage virtualization is not a new concept. The concept of taking many physical disk drives and representing them by volume names or drive letters began with the introduction of RAID arrays in the late 1970s. And the virtualization debate revived with the advent of architectures for network storage, such as Storage Area Network (SAN). In the case of storage virtualization, a virtual volume refers to a potentially large number of physical disk drives or other disk-based storage such as array partitions. Array partitions are self-created virtual volumes on a subset of the disks in the array. In the case of a storage network, almost anything that can be expressed as a logical unit number (LUN) can be aggregated into a virtual volume.

The tremendous trend of increasing storage use has put a lot of pressure on storage operations and data management on a daily basis. As a result, satisfying service levels for availability and provisioning is a major challenge. To ease this burden, companies have begun to turn to disk and tape storage virtualization technologies. Storage virtualization is an integral part of an on demand strategy, allowing changes to the hardware infrastructure to be made with little or no impact on applications. As a result, storage virtualization facilitates management, increases application availability, and lowers total cost of ownership. Figure 3 shows a typical configuration and conceptual diagram for server and network virtualization.

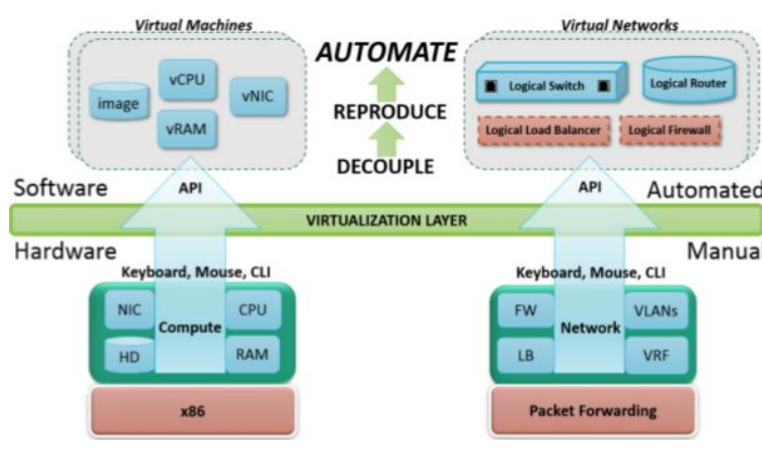

Fig. 3. Server and network virtualization concept

How virtualization works is that software called a hypervisor separates physical resources from a virtual environment that requires them. Hypervisors can be deployed on operating systems such as laptops or installed directly on hardware such as servers, and most companies virtualize this way. The hypervisor partitions the physical resources and makes them available in a virtual environment. Resources are partitioned from physical to virtual environments as needed. Users interact with the virtual environment (commonly referred to as guest machines or virtual machines) and run calculations within the virtual environment. A virtual machine functions like a single data file. Just like digital files, you can move them from one computer to another and open them on either computer and work the same. When a virtual environment is running and a user or program issues a command that requires additional resources from the physical environment, the hypervisor passes the request to the physical system and caches the changes. All of this is done near native speed.

After all, in order to provide a Smart work service in a mobile environment, security problems must be solved first, so research and application of mobile virtualization technology must be preceded. Therefore, it is necessary to secure and apply a technology for operating the latest operating system based on mobile virtualization technology and storage virtualization technology (mobile cloud resource management technology), which is the core of mobile security.

In the paper, a dynamic service application structure was proposed for Smart work service applying virtualization-based security technology. A service change request can be made while the mobile device is operating a service, and the changed service can provide the changed service not only to the existing user of the Smart work system but also to other device users. As such, this study provides an adaptive architecture that can provide dynamic services beyond the static service range of the existing Smart work system to flexibly respond to 
various changes in companies. Figure 4 shows the structure of a dynamic service type Smart work system.

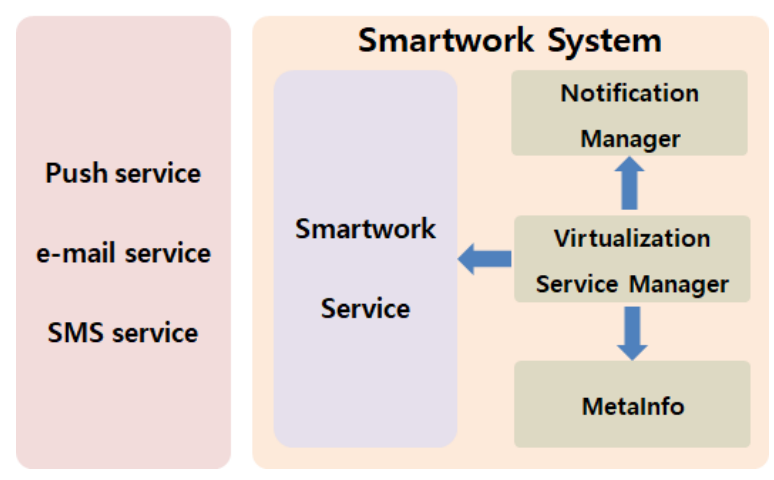

Fig. 4. Smart work system architecture

Unlike the existing service method, the Smart work service is not managed by a single channel for service settings, but can provide a system that can change and manage services anytime, anywhere through multi-channel. The Smart work service process starts when an administrator requests a service change. Meta information is changed by the adaptive service manager in the Smart work system after a service change request. The changed service is notified to users who use the service through the notification service. Finally, users who use the service can use the changed service without stopping the service.

Finally, the platform structure consists of systems such as web conferencing, collaboration tools, mobile offices, business apps, and security for small and medium-sized businesses based on an open platform and infrastructure such as internet service cloud of telecommunication companies. It is a system that provides an environment in which Smart workers can receive services centered on users according to the Smart worker field by the Smart work floor.

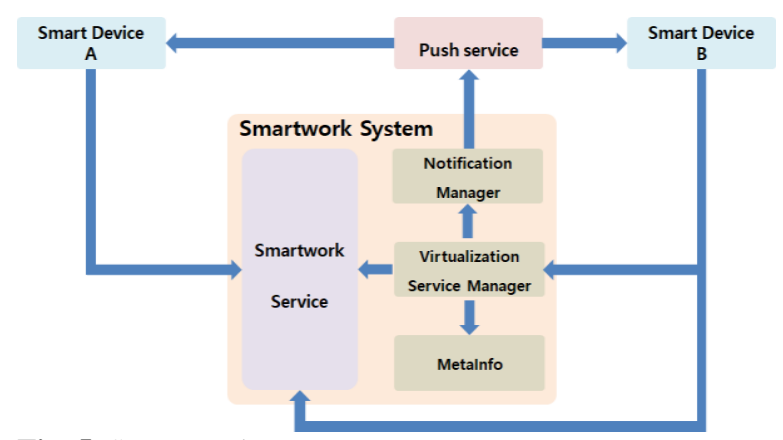

Fig. 5. Smart work process

\section{Conclusion}

Due to the recent development of the 5th generation mobile communication and the growth of smart phones that provide rich mobile applications utilizing the same, various services are used through smart phones. In addition, the non-face-to-face industry is revitalizing due to corona, and non-face-to-face business processing based on various services is rapidly increasing. However, the advantages of the smartphone's convenience are achieved through 'proactive exposure' of personal information such as one's location information, gender, and job, and there is an aspect of focusing information to increase productivity.

Smartphone threats can be broadly divided into loss, malware infection, information leakage, financial loss, and exploitation of attack sites. It can be a threat to users, telecommunications service providers, terminal manufacturers, and information providers, but it has the most damage to users because it is a structure that causes leakage of personal information and financial loss, and measures are needed. In particular, if you lose your smartphone, you may suffer irreparable losses. Therefore, a security enhancement technology for important data or files is required, and this paper proposes a Smart work service applying a virtualization-based security technology for protecting important information in such a mobile environment.

In this paper, in order to overcome the limitations and problems of mobile security, we designed a Smart work service that can operate on various mobile devices such as smartphones and tablet PCs by fusion of mobile security technology and mobile virtualization technology. In addition, a Smart work service based on mobile virtualization that can be operated on the latest Android and Linux operating systems was proposed.

By providing a virtualization function for a device in a mobile device, it is possible to efficiently use the resources in the device. In addition, by separating the execution environment of the terminal into two domains and performing the storage and security functions of important data in the safety domain, it is possible to fundamentally block the attempt to leak important data through routing in the general domain. Since the cryptographic functions in the security engine include SEED and ARIA, which are domestic cryptographic algorithms, it is possible to interwork with existing domestic security systems. In addition, it is possible to provide various security services required in a user-centered mobile terminal environment, and can be used as a base technology for mobile security solutions such as MDM.

The storage virtualization-based mobile work service proposed in this paper can be used for mobile terminals and special security-enhanced terminals 
that require high security in mobile environments. In addition, it can be used for mobile financial services for safe payment/payment such as electronic payment and internet banking, and prevents corporate information leakage and illegal access by unauthorized users in work environments such as Smart work using mobile terminals. It can be used to ensure the safety of corporate cloud services. In the future, it is expected to be used as an important security function in establishing mobile egovernment such as administrative civil service and on-site support service.

\section{REFERENCES}

[1] Zheng, P. \& Lionel N. (2006). Introduction to Smart Phone and Mobile Computing. Smart Phone and Next Generation Mobile Computing, 1-21.

[2] Jiaqi, W., Yanxiang, G., Wenhan, H., Jianbo, Z., Hong, P., Xiping, H., \& Cheng, J. (2019). Mobile crowdsourcing based context-aware smart alarm sound for smart living. Pervasive and Mobile Computing, 55, 32-44.

[3] Yuzhong, Z., \& Chongwen, W. (2016). Product Images Retrieval Based on Smart Mobile Devices. International Journal of Computer Theory and Engineering, 8(5), 403-408.

[4] Ahn, J. U., \& Lee, B. M. (2020). Smart Edge Broker for Location-Based Transfer between Services and Distributed Data in IoT Smart Services. Mobile Information Systems, 2020, 1-12.

[5] Leo Bhebhe, \& Rauli Parkkali. (2011). VoIP Performance over HSPA with Different VoIP Clients. Wireless Personal Communications, 58(3), 613-626.

[6] Jaclyn, P., \& William, R. (2018). VoIP Accessibility: A Usability Study of Voice over Internet Protocol (VoIP) Systems and a Survey of VoIP Users with Vision Loss. Journal of Visual Impairment \& Blindness, 112(1), 47-60.

[7] Guozhong, L. (2019). An Introduction to the Mobile Payment Market and Trend in China. International Journal of Emerging Multidisciplinary Research, 3(1), $1-5$.

[8] Rachel Lara Cohen. (2010). Rethinking mobile work: boundaries of space, time and social relation in the working lives of mobile hairstylists. Work, Employment and Society, 24(1), 65-84.

[9] Karlene, C., \& Daniel, R. (2015). Managing work-life boundaries with mobile technologies. Information Technology \& People, 28(1), 34-71.

[10] Townsend, K., \& Batchelor, L. (2005). Managing mobile phones: a work/non-work collision in small business. New Technology, Work and Employment, 20(3), 259-267.

[11] Hong, S. H., Park, S. H., \& Noe Lopez-Benitez. (2017). Trends and Implications of Mobile and Online FinTech. International Journal of Emerging Multidisciplinary Research, 1(1), 43-47.

[12] Shin, H. J., Jeon, M. H. \& Oh, C. H. (2018). DEVELOPMENT OF SMART WORK-BASED PRODUCTION MANAGEMENT SYSTEM TO
IMPROVE WORK EFFICIENCY IN SMART FACTORY. Far East Journal of Electronics and Communications, 18(1), 101-111.

[13] Keunwoo, R., Woongryul, J., \& Dongho, W. (2012). Security Requirements of a Mobile Device Management System. International Journal of Security and its Applications, 6(2), 353-358.

[14] Ronald, P., Leendert, V.D., \& Reiner, S. (2008). Virtualization and Hardware-Based Security. IEEE Security and Privacy, 6(5), 24-31.

[15] Paul, B., Boris, D., Keir, F., Steven, H., Tim, H., \& Alex, H. (2003). Xen and the Art of Virtualization. Proceedings of the nineteenth ACM symposium on Operating systems principles, 164-77.

[16] Durairaj, M., \& Manimaran, A. (2015). A Study on Security Issues in Cloud based E-Learning. Indian Journal of Science and Technology, 8(8), 757-765.

[17] Jae-Deok, L., \& Jeong-Nyeo, K. (2014). A Study on the Trusted App.- based Access Control to the Isolated Trusted Execution Environment in Mobile Device. Proceedings of the Korean Institute of Communications and Information Sciences Conference, Korea, 364-365.

[18] Seehwan, Y., \& Chuck, Y. (2014). Real-Time Scheduling for XenARM Virtual Machines. IEEE Transactions on Mobile Computing, 13(8), 18571867.

[19] Hwi-Min, C., Chang-Bok, J., \& Joo-Man, K. (2014). Efficient security method using mobile virtualization technology and trustzone of ARM. Journal of Digital Convergence, 12(10), 299-308. 